\title{
A vigilância alimentar e nutricional em 20 anos da Política Nacional de Alimentação e Nutrição
}

\author{
Food and nutrition surveillance in 20 years of the \\ Brazilian National Food and Nutrition Policy
}

\author{
Vigilancia alimentaria y nutricional en los 20 años \\ de la Política Nacional de Alimentación \\ y Nutrición
}

Daniela Souzalima Campos 1,2

Patrícia Costa Fonseca 3,4

doi: 10.1590/0102-311X00045821

Para o Ministério da Saúde 1, a avaliação contínua do perfil alimentar e nutricional da população e seus fatores determinantes compõe a vigilância alimentar e nutricional, diretriz essencial para a atenção nutricional no Sistema Único de Saúde (SUS), para organização e gestão dos cuidados em alimentação e nutrição na Rede de Atenção à Saúde. Essa organização deve iniciar-se pelo reconhecimento da situação alimentar e nutricional da população adstrita aos serviços e às equipes de atenção primária à saúde (APS) 1, somado a informações provenientes de outras fontes, como pesquisas, inquéritos populacionais e outros Sistemas de Informações em Saúde (SIS), para subsidiar a construção e avaliação de políticas públicas coerentes com as necessidades locais.

O Sistema de Vigilância Alimentar e Nutricional (SISVAN) promove informação contínua sobre o estado alimentar e nutricional da população acompanhada. Esses dados são valiosos para traçar o perfil epidemiológico e nutricional e refletem o impacto das ações realizadas em determinadas comunidades 2. Por muitos anos, tais dados foram coletados e sistematizados pelos estados por métodos diferentes, inviabilizando uma análise da situação alimentar e nutricional da população assistida no SUS. O sistema informatizado como se conhece hoje surgiu em dezembro de 2007, com a incorporação das curvas de crescimento da Organização Mundial da Saúde (2006/2007) para a avaliação do estado nutricional de crianças e adolescentes. O sistema oficial de informação permitiu, primeiramente, a inserção de dados antropométricos para todos os ciclos etários, e mais tarde de consumo alimentar. O lançamento do sistema foi uma oportunidade de qualificação dos profissionais para coleta de dados, avaliação nutricional, registro e fomento do uso da informação para tomada de decisão.

O SISVAN gera informações contínuas e úteis para o uso imediato - informação para a ação - tanto para os gestores, no planejamento dos planos de ação, quanto para os profissionais, na reorganização do atendimento nutricional na unidade de saúde. No entanto, observa-se que as informações não têm sido utilizadas em seu potencial para o planejamento, gestão e avaliação de ações de alimentação e nutrição no âmbito da atenção primária à saúde 3,4 e, consequentemente, pouco contribuem para implementação da Política Nacional de Alimentação e Nutrição (PNAN). Nesse sentido, evidenciase a necessidade de investir em qualificação de profissionais e gestores para melhor utilização desse sistema, e ampliar a divulgação de informações geradas por ele, de forma clara, tanto para a população e os conselhos quanto para os profissionais de saúde e outros setores como agricultura, assistência social e educação. Materiais como boletins, mapas, informativos podem ser produzidos e amplamen-
1 Secretaria de Estado de Saúde de Minas Gerais, Belo Horizonte, Brasil. 2 Universidade Federal de Minas Gerais, Belo Horizonte, Brasil. 3 Superintendência Regional de Saúde de Pouso Alegre, Pouso Alegre, Brasil.

4 Centro Universitário UNA, Pouso Alegre, Brasil.

\section{Correspondência}

D. S. Campos Rua Barão de Saramenha 472, apto. 401, Belo Horizonte, $M G$ 31010-490, Brasil.

daniela.campos@saude.mg.gov.br 
te divulgados na própria unidade de saúde, pelas informações da própria região e/ou de forma mais abrangente, para sensibilizar e informar a população, profissionais de saúde, gestores, entre outros.

Outra medida que pode contribuir para maior utilização das informações geradas pelo SISVAN é a revisão dos relatórios por ele emitidos. O sistema precisa avançar para permitir diferentes análises, como por exemplo, conhecer a relação entre o estado nutricional, o consumo alimentar e os determinantes sociais de saúde (raça, etnia, sexo, orientação sexual, renda, escolaridade, povos e comunidades tradicionais, entre outras).

Outro ponto abordado no artigo de Santos et al. 5, é a tendência de aumento na cobertura do SISVAN, apesar de ainda apresentar baixos percentuais. As maiores cobertura estão entre gestantes, crianças e adolescentes 6 , dada a vinculação de indicadores de avaliação do estado nutricional ao repasse financeiro aos municípios, como ocorre com o Programa Bolsa Família, o Programa Saúde na Escola e o Crescer Saudável. Se, por um lado, a estratégia promove o aumento da cobertura, por outro, contribui para uma percepção do caráter burocrático de alimentação do sistema, e, limita a vigilância alimentar e nutricional a determinados ciclos etários e apenas ao subsistema nutricional. Desse modo, seria positivo se os indicadores vinculados a recursos financeiros fossem mais abrangentes quanto ao público, incluíssem marcadores de consumo alimentar e apresentassem como metas não apenas a cobertura populacional, mas o monitoramento da evolução de agravos nutricionais e do consumo de alimentos saudáveis e não saudáveis.

Mais recentemente, um importante avanço foi a integração do SISVAN à Estratégia e-SUS Atenção Básica (e-SUS AB) e ao Sistema de Gestão do Programa Bolsa Família na Saúde, que ocorreu a partir de 2016. A integração dos diversos SIS no âmbito da APS é considerada como uma qualificação da gestão da informação, essencial para ampliar a qualidade no atendimento à população 7 . Na prática, essa medida reduz retrabalho dos profissionais de saúde e aumenta a cobertura populacional, tendo em vista que a avaliação nutricional faz parte da rotina de muitos atendimentos da APS. Ainda em relação à integração de sistemas, um avanço desejável é a integração com a rede de saúde suplementar, para aumentar a representatividade das informações na população brasileira.

Nesses 20 anos da PNAN, destaca-se a publicação de materiais educativos, manuais técnicos e cursos de formação e qualificação profissional relacionados à vigilância alimentar e nutricional pelo MS, fundamentais para apoiar a organização da assistência ofertada a população. Muitos desses, produzidos em parceria com instituições de ensino, o que amplia a qualificação e a divulgação dos materiais. Dentre essas, destacam-se: Protocolos do Sistema de Vigilância Alimentar e Nutricional - SISVAN na Assistência à Saúde 8, de 2008, que objetiva sistematizar a avaliação do estado nutricional do público que busca atendimento na rede básica de saúde, e Orientações para Coleta e Análise de Dados Antropométricos em Serviços de Saúde: Norma Técnica do Sistema de Vigilância Alimentar e Nutricional - SISVAN 9, de 2011, que traz informações básicas sobre antropometria, visando à coleta de informações necessárias para a realização da Vigilância Nutricional entre indivíduos em diferentes fases do curso da vida.

Uma estratégia importante foi a criação de incentivo financeiro exclusivo para aquisição de equipamentos antropométricos para as unidades básicas de saúde (UBS), por meio da Portaria no 2.975/2011. Entre 2011 e 2018, foram publicadas outras 11 portarias homologando municípios ao recebimento desse recurso. O incentivo foi acompanhado do manual orientador para aquisição de equipamentos antropométricos para orientar os municípios quanto à descrição correta de equipamentos adequados à vigilância alimentar e nutricional, como antropômetros, balanças e fita métrica.

$\mathrm{O}$ fomento à pesquisa e à realização periódica de inquéritos populacionais é uma das estratégias da vigilância alimentar e nutricional. Desde a publicação da primeira versão da PNAN, observa-se a realização de relevantes inquéritos, como a Pesquisa Nacional de Demografia e Saúde (PNDS), a Pesquisa de Orçamentos Familiares (POF) que, em algumas de suas edições, apresentou os módulos de consumo alimentar e/ou de antropometria, a Pesquisa de Avaliação do Impacto da Iodação do Sal (PNAISAL), a Pesquisa Nacional de Saúde do Escolar (PeNSE), o Estudo de Riscos Cardiovasculares em Adolescentes (ERICA), a Vigilância dos Fatores de Risco e Proteção para Doenças Crônicas por Inquérito Telefônico (VIGITEL) e, recentemente, o Estudo Nacional de Alimentação e Nutrição Infantil (ENANI). A análise desses inquéritos nacionais permitiu constatar mudanças no padrão alimentar da população brasileira, tendência à redução da desnutrição e aumento da obesidade. Os inquéritos permitem elucidar os fatores associados, características e consequências dos problemas nutricionais que ainda afetam vastos segmentos 
populacionais nas diversas regiões brasileiras e, portanto, permitem subsidiar as intervenções para uma melhor qualidade de vida da população 2,10,11.

Notam-se, da mesma forma, avanços na pesquisa científica. Uma busca simples em base de dados BIREME mostra que cresce o número de artigos localizados com o descritor vigilância alimentar e nutricional. Evidencia-se o esforço em fomentar pesquisas relacionadas à vigilância alimentar e nutricional e progressivo interesse da comunidade científica nacional e internacional pelo tema, que amplia a contribuição da ciência na proposição de reflexões e soluções de problemas relacionados à vigilância alimentar e nutricional.

Entre os desafios para o fortalecimento da vigilância alimentar e nutricional no cuidado nutricional, apontamos a importância de fomentar o uso dos relatórios do SISVAN, dos inquéritos populacionais e pesquisas, pelos profissionais de saúde e pelos gestores formuladores de políticas públicas do SUS, tanto para planejamento de ações de alimentação e nutrição quanto para avaliação dos programas e ações existentes em nível local, municipal, estadual e federal. Diante do apresentado, pode-se afirmar que foram vivenciados importantes avanços relacionados à vigilância alimentar e nutricional nos últimos 20 anos no Brasil. Ainda restam desafios a superar para que a vigilância alimentar e nutricional se fortaleça como pedra fundamental para planejamento e avaliação de políticas públicas focadas na melhoria da situação alimentar e nutricional da população.

\section{Colaboradores}

D. S. Campos e P. C. Fonseca participaram igualmente da produção do texto.

\section{Informações adicionais}

ORCID: Daniela Souzalima Campos (0000-00033234-9939); Patrícia Costa Fonseca (0000-00033760-8581).
1. Departamento de Atenção Básica, Secretaria de Atenção à Saúde, Ministério da Saúde. Marco de referência da vigilância alimentar e nutricional. Brasília: Ministério da Saúde; 2015.

2. Coutinho JG, Cardoso AJC, Toral N, Silva ACS, Ubarana JA, Aquino KKN, et al. A organização da vigilância alimentar e nutricional no Sistema Único de Saúde: histórico e desafios atuais. Rev Bras Epidemiol 2009; 12:688-99.

3. Rolim MD, Lima SML, Barros DC, Andrade CLT. Avaliação do SISVAN na gestão de ações de alimentação e nutrição em Minas Gerais, Brasil. Ciênc Saúde Colet 2015; 20:2359-69.

4. Vitorino SAS, Cruz MM, Barros DC. Validação do modelo lógico teórico da vigilância alimentar e nutricional na atenção primária em saúde, Cad Saúde Pública 2017; 33:e000147217.

5. Santos SMC, Ramos FP, Medeiros MAT, Mata MM, Vasconcelos FAG. Avanços e desafios nos 20 anos da Política Nacional de Alimentação e Nutrição. Cad Saúde Pública 2021; 37 Suppl 1:e00150220. 
6. Nascimento FA, Silva SA, Jaime PC. Cobertura da avaliação do estado nutricional no Sistema de Vigilância Alimentar e Nutricional brasileiro: 2008 a 2013. Cad Saúde Pública 2017; 33:e00161516.

7. Vitorino SAS, Siqueira RL, Passos MC, Bezerra OMPA, Cruz MM, Silva CAM. Estrutura da vigilância alimentar e nutricional na atenção básica em saúde no Estado de Minas Gerais. Rev APS 2016; 19:230-44.

8. Ministério da Saúde. Protocolos do Sistema de Vigilância Alimentar e Nutricional - SISVAN na assistência à saúde. Brasília: Ministério da Saúde; 2008. (Série B. Textos Básicos de Saúde).

9. Ministério da Saúde. Orientações para coleta e análise de dados antropométricos em serviços de saúde: norma técnica do Sistema de Vigilância Alimentar e Nutricional - SISVAN. Brasília: Ministério da Saúde; 2011. (Série G. Estatística e Informação em Saúde).
10. Sperandio N, Priore SE. Inquéritos antropométricos e alimentares na população brasileira: importante fonte de dados para o desenvolvimento de pesquisas. Ciênc Saúde Colet 2017; 22:499-508.

11. Universidade Federal do Rio de Janeiro. Estudo Nacional de Alimentação e Nutrição Infantil - ENANI-2019: resultados preliminares. Indicadores de aleitamento materno no Brasil, 2020. Rio de Janeiro: Universidade Federal do Rio de Janeiro; 2020. 